\title{
In-Line Dynamic Acoustic Behavior of a Viscoelastic Complex Media: Dough Application
}

\author{
G. Nassar*, B. Nongaillard and J. Chéio
}

I.E.M.N.-U.M.R. C.N.R.S. 8520-D.O.A.E.-Université de Valenciennes 59313 Valenciennes Cedex 09, France

\begin{abstract}
The main objective of this study was to understand the physical law characterizing dough during kneading. Throughout the process, dough is subjected to extensional deformation resulting from the mechanical component and the development of holes in the matrix. For that, we developed a very low frequency acoustic device $(<100 \mathrm{~Hz})$ to follow the mechanical evolution of the dough through an in-line acoustic sensor with part of the mixer playing the role of an acoustic receiver (ear-phone), which exploits the background noise of the process. Depending on the physical state of the product, acoustic amplitude of sound was chosen to represent the evolution of the matter. Via a signal processing modulus this sensor shows the mechanical progress of the dough by means of a curve with critical points. The results show that the acoustic component is able to follow the evolution of the physical properties of the matter in situ. The results using various technological parameters indicate that a high degree of sensitivity can be reached with non-destructive acoustic techniques in highly absorbing media.
\end{abstract}

Keywords: Acoustic, noise, dough, kinetic.

\section{INTRODUCTION}

With the progress in technology, complex media have become more and more present in research and industry. Such physical properties cover a wide variety of areas as in medical or bio-medical domain (osteopathy) or in agroindustrial product and process (e.g. pharmaceutics, cosmetic,...). Some work has contributed to the understanding of specific scientific problems, which in the majority of cases are based on mathematical or numerical models.

It is important to note that the propagation of sound in a heterogeneous and porous environment is often very difficult. This complexity becomes a characteristic when the matter has some viscoelastic properties.

By using the acoustics experimental way, this work focuses mainly on the development of a viscoelastic evolving matter according to several technological parameters. For that, the dough-making process was chosen due to the complexity of such product like a heterogeneous and aggregate medium produced by mixing the raw material at the beginning of process.

Quantitative understanding of the physical mechanisms is still very limited for such matter. One of the possible reasons for this is that several fundamental complex physical processes are involved. As the texture changes and the volume increases, the dough is subjected to both shear and extensional deformations. It can be noted that the underlying

*Address correspondence to this author at the I.E.M.N.-U.M.R. C.N.R.S. 8520-D.O.A.E.-Université de Valenciennes 59313 Valenciennes Cedex 09, France; Tel: 333275114 47; Fax: 33327511189 ;

E-mail: gnassar@univ-valenciennes.fr processes involved in modern technologies such as continuous processes and the chemical or biological mechanism improves used can only be determined from detailed knowledge of the mechanical phenomena occurring through an understanding of the dynamic viscoelastic and physical structure of the matter.

In addition, the complexity of dough is not restricted to its chemical composition, but also includes physical properties [1-4]. Despite considerable changes in the physical properties during kneading, there are few studies in the literature concerning the transition step [5]. From a structural point of view, evolving dough can be considered as a porous material consisting of gas cells embedded in a viscoelastic matrix. Generally, rheological tests have been used to follow the changes in the fundamental mechanical properties [6-8]. Some other works [9-11] describe the ability of the viscoelastic nature of the matrix to retain gas. In order to obtain some information on the physical changes during the formation of this type of product, models have been studied and showed that dynamic methods are useful for understanding viscoelastic changes [12].

In addition, some works have been published that deal with different techniques, chemical or physical. Ultrasonic techniques are widely used for the characterization of materials. With their non-destructive nature, the interest lies in their great sensitivity to the physical properties of the evolving medium studied [13], which is an important advantage. The only disadvantage is that when the structure of the medium studied is complex and heterogeneous, like dough for example, the propagation of the ultrasonic wave becomes very difficult due to attenuation and scattering phenomena. 
Many authors have used ultrasound to characterize dough [14-19]. However, in the majority of these studies, the measurements were carried out on static matter (dough without yeast) or on samples of small thicknesses due to the complexity of this medium and the strong wave absorption it induces.

At present, it is difficult to measure this phenomenon directly. So, with the aim of understanding this complex phenomenon, the acoustic noises generated by the process were taken as relevant information to define the critical steps of evolving matter.

In this paper, we present two acoustic techniques to follow the physical state of such evolving matter according to several technological parameters. With this aim in mind, for the structural phase (mixing phase) we propose a passive measurement based on the use of the amplitude variation given by the background noise generated by the process.

\section{STRUCTURAL PHASE: FROM A GRANULAR MIXTURE TO A VISCO-ELASTO-PLASTIC MATTER}

\subsection{The Function of Mixing}

In the bread-making process, time, shape and work during the mixing phase play a major role in the formation and the development of the gluten network in the dough and the incorporation of gas bubbles.

The key stages taking place during kneading can be summarized as follows:

1. The uniform dispersion of the raw material.

2. Dissolution and hydration of that material, in particular the flour proteins and the damaged starch.

3. The development of a gluten (hydrated flour protein) structure in the dough arising from the input of mechanical energy from the mixing action.

4. The incorporation of air bubbles within the dough to provide the gas bubble nuclei for the carbon dioxide will be generated by yeast fermentation and oxygen for oxidation and yeast activity.

5. The formation of dough with suitable rheological properties for subsequent processing.

It can be noted that the size and number of gas bubbles in the dough at the end of mixing are strongly influenced by the mechanism of dough formation and the mixing conditions.

We know that gluten is formed by bonds between glutenin and gliadin.

In the formation of dough, these bonds are formed mechanically by kneading, then chemically with mixtures of oxidants or reductants, or by fermentation.

\subsection{Effect of Work on the Mechanical Properties of the Matter}

Under the mechanical action during kneading, the flour is transformed into a viscoelastic substance composed of two non-miscible continuous aqueous phases: an elastic phase represented by the gluten network which encloses a liquid phase containing suspended starch granules $(\sim 10 \mu \mathrm{m}$ in size) and other hydro-soluble compounds [20].

This action also forms an ordered cohesive mass. Kneading leads to a very uniform mass in which very extensive gluten formation (extensive, homogenous crosslinking) produces a strong substance which will rise spectacularly and have good mechanical strength (Fig. 1).

Excess kneading can damage the gluten network, increase protein solubility and decrease the extractability of the lipids [21], thus resulting in sticky dough [22]. This phenomenon is due to the fact that the mechanical forces applied to the matter decrease, by mechanically breaking, the length of the chains and thus the molecular weight of the proteins.

\section{MATERIALS AND METHODS}

The use of acoustic measurements was tested to monitor the aggregation phenomenon during the dough mixing process. This approach exploits the noise produced by the process to monitor the progress of the formation of the dough.

The advantage of this technique is the possibility to exploit a broad frequency spectrum of a given acoustic source generated by the process itself; background noise. This noise previously considered detrimental for acoustic applications, is of the greatest importance for our application.

The components of this "useful" acoustic noise will depend on the evolution of physical properties of the product.

However, the physical choice (size, nature, ...) of the sensor and its integration will act as a filter for some frequency components. In the kneading phase, this choice favors low frequencies that reflect, after analysis, the evolution of mechanical properties of the dough.

The composition of the samples used in the basic formulation is presented in Table $\mathbf{1}$.

Using the Alveograph NG Chopin technique in first time, the formation of the dough matrix was analyzed in-line with a piezoelectric sensor, favoring the shear component, embedded in the planar zone of the kneading tank (Fig. 2). This sensor is $20 \mathrm{~mm}$ in diameter and $2 \mathrm{~mm}$ thick. It is used as a microphone which captures the noise from the movement of the dough that is continuously turned by the blade. Each time the blade passes in front of the sensor, an electric signal reflecting the structural state of the dough at that moment is sent. After digitalization, the signals are processed on a computer for a better understanding of the noise emanating from the mixer. The acoustic signal is routed by a GPIB (National Instrument) protocol to our prototype to be collected and analyzed.

It can be noted that the electrical management of the measuring system, the signal acquisition and the signal processing are controlled by LabView software with a signal acquisition frequency of $2 \mathrm{~s}$.

The mixer blends the dough using a rotating flat blade with a previously controlled torque and temperature 


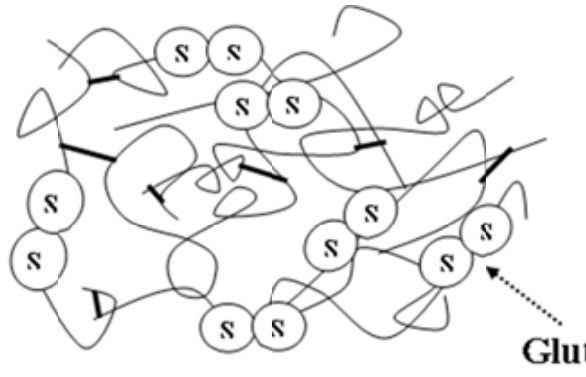

\section{Glutenin chain with intermolecular} covalent disulfide bonds

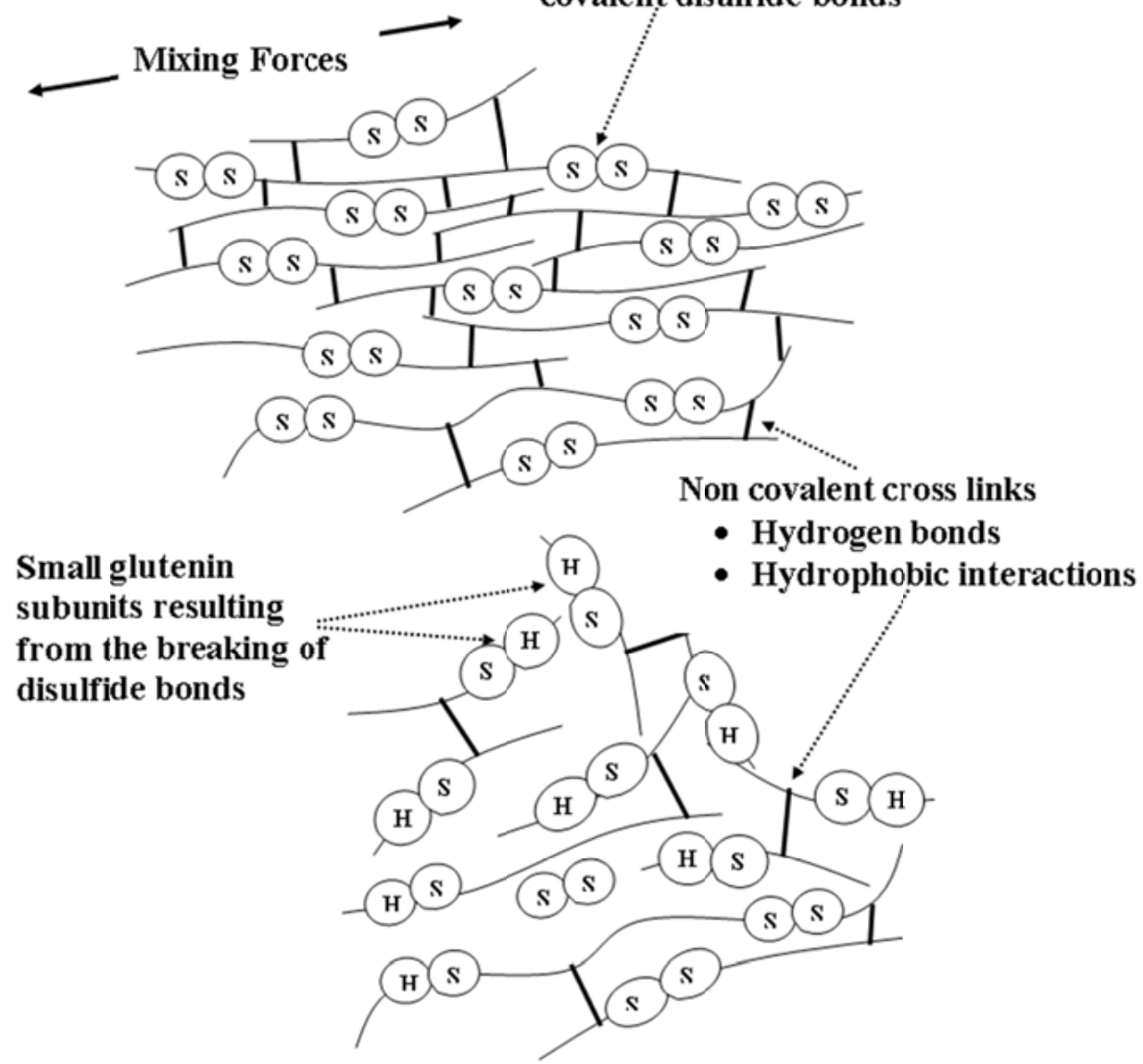

Fig. (1). A model for glutenin hyper-aggregation; Molecular interpretation of gluten development: (a) start of mixing, (b) optimum formation, (c) over-mixing (Létang et al., 1999).

$\left(25 \pm 0.5^{\circ} \mathrm{C}\right)$. Using this technique, the kneading time to reach the maturation of the matter is about 15 minutes. The sample preparation protocol consists of mixing the dry ingredients in the mixer for one minute, then adding the water without stopping the rotation of the blade.

Table 1. Composition Ratio of Dough Components (Reference)

\begin{tabular}{|c|c|c|c|}
\hline Component & Baker Flour (T55) & Water & Salt \\
\hline \hline \% of Flour mass & $100 \%$ & $60 \%$ & $2 \%$ \\
\hline Mass $(\mathrm{g})$ & $200 \mathrm{~g}$ & $120 \mathrm{~g}$ & $4 \mathrm{~g}$ \\
\hline
\end{tabular}

\subsection{Preliminary Results}

In order to understand the phenomena governing dough formation in the kneading phase, two types of flour were used: soft and strong flour versus the moisture and salt content.

Wheat flour contains different particle fractions. In our case, we allocated the name "soft flour" to the product containing carbohydrates $(72 \%)$, protein $(13 \%)$, lipids $(1.5$ $\%)$, moisture (14\%) and non starch polysaccharides $(2-3 \%)$, all expressed as percentage dry weight.

"Strong flour" differs from the weak one by the protein content which can reach $18-20 \%$. Fig. (3) shows, through the variation in amplitude of the acoustic vibration, the progress of the formation inside the cell of two standard composition dough samples at constant temperature $\left(25^{\circ} \mathrm{C}\right)$; soft and strong flour.

Fig. (3) shows the evolution of the maximum amplitude of the signal received by the sensor embedded in the wall of the mixer. It can be noted that the curves start at a specific value and reach a maximum value; these values vary 


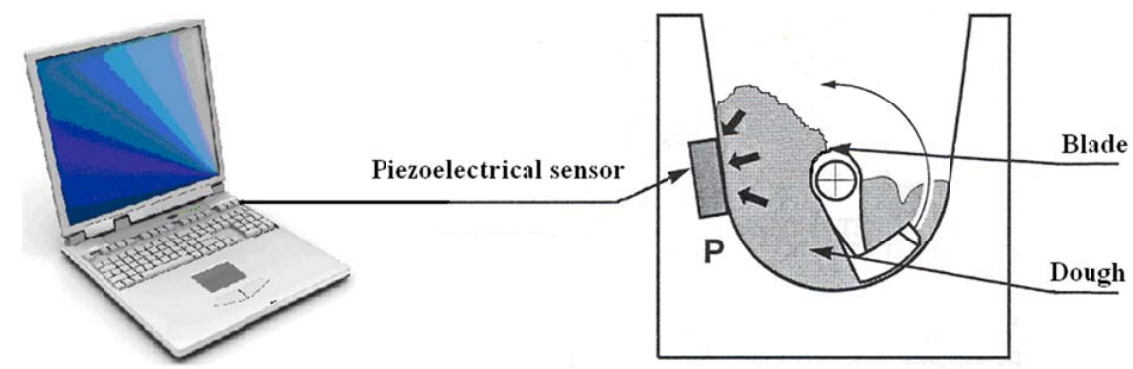

Fig. (2). Position of measurement device in mixer cell using the Alveograph NG Chopin.

according to the type of flour used. In the experiment considered, the strong flour starts with an amplitude level higher than that of the soft flour. This phenomenon reflects a medium having a greater mechanical resistance at the start. This mixture reaches optimum maturity with a lead about 3 minutes compared to that made by soft flour. These results, which are in agreement with the literature [23, 24], indicate a critical transition phase (the max index) defining the optimal formation of the dough.

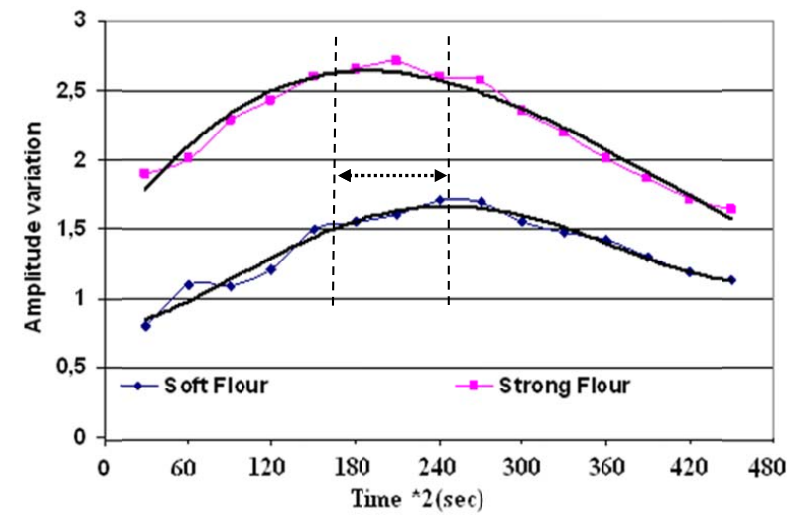

Fig. (3). Evolution in acoustic properties for two kinds of flour tested.

The results indicated that the increase in the power drawn by the mixer motor is due to the increase in both apparent shear and extensional viscosities.

By using the acoustic measurement technique for samples with different water content, we can see that from the "Max" value (maximum on the curves) and the position of the "Max" (Index) there is an optimal relationship between the various components of dough. A variation in one of these components gives underdeveloped dough in the case of lack of water or a very sticky product in the case of excess water (Fig. 4).

\subsection{Opinion}

This application shows the potential of an acoustic method to characterize the dough-making process. This technique used an embedded acoustic sensor in the kneading cell, working in a passive position and exploiting the mechanical noise emitted by the mixer. The measurements can be used to determine one critical point of dough formation in the kneading phase. We have shown that the signals received using this technique evolves differently according to the properties of the product used: soft and strong flour. The results have shown that this technique can be used as an effective in-line control method in the kneading process.

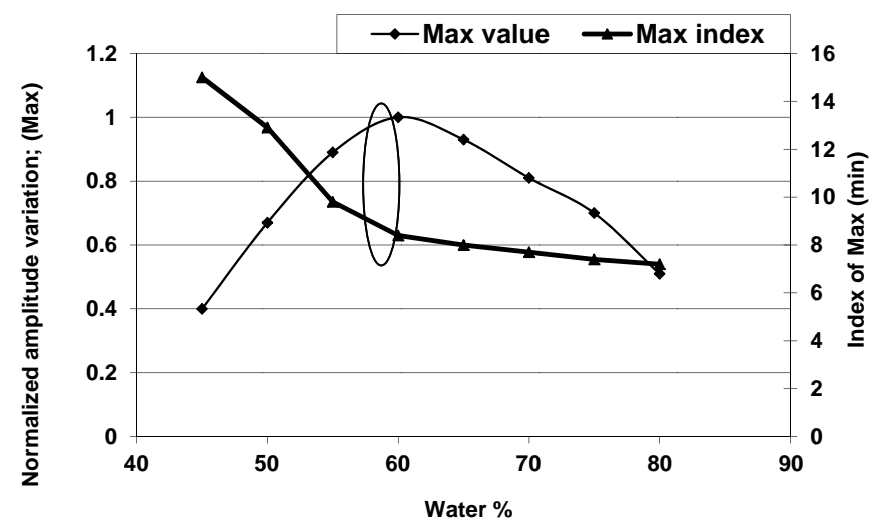

Fig. (4). Acoustical properties evolution of kneading phenomenon showing the optimal ratio of raw leading to an optimal maturation.

However, the lack of precision on the curve obtained (Fig. 3) and consequently the lack of detailed analysis of the process led us to rely on another technique of which the sensor is an integral part of the mixer.

\section{IN-LINE MEASUREMENT: SPI 10 VMI MIXER}

\subsection{Acoustic Device}

Until now, problems encountered with fundamental tests include: sample preparation; instrumentation, which requires a delicate set up in a humid or dusty atmosphere and is difficult to maintain in an industrial environment as well as requiring high levels of technical skill; often inappropriate deformation conditions; difficulty in interpreting the results and slip and edge effects during testing, hence the interest in direct measurement without any special precautions.

Using the SPI 10 (VMI, Montaigu, France) mixer technique, the formation of the dough matrix was analyzed in-line with an acoustic sensor made of a piezoelectric plate element embedded in the central mixer rod (Fig. 5). The dimension of the PZT active element was optimized for a plate mode resonance according to the frequency band resonance of the rod whose fundamental $\left(1^{\text {st }}\right)$ mode is about $30 \mathrm{~Hz}$.

This rod, which plays the role of a very low frequency ear-phone, is used as a microphone that captures the noise from the movement of the dough that is continuously turned in the mixer. Each time the rod is solicited, its vibratory 
properties are converted into an electric signal reflecting instantaneously the structural state of the matter being tested (Fig. 6). After digitalization, the signals are processed on a computer for a better understanding of the noise emanating from the mixer.
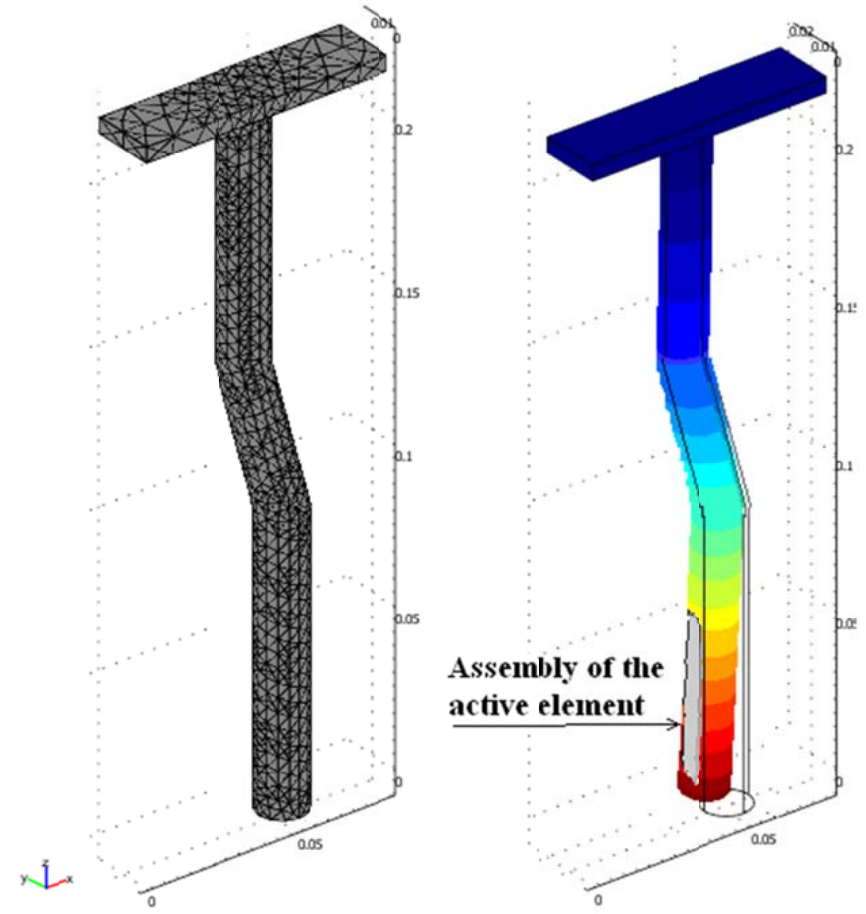

Fig. (5). Fundamental mode of rod vibration: a very low frequency acoustic sensor $(30 \mathrm{~Hz})$.

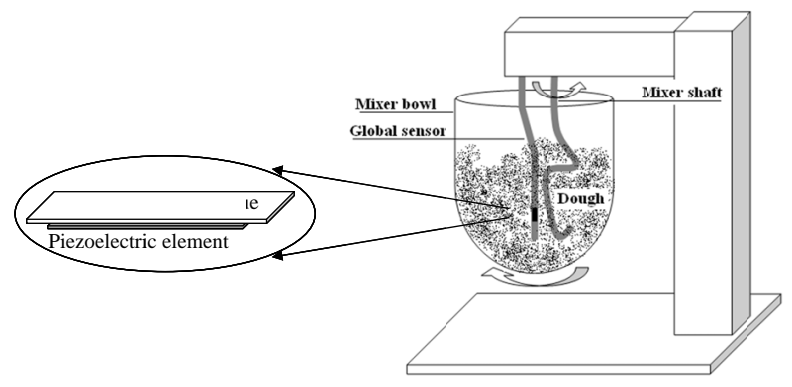

Fig. (6). Position of measurement device in mixer cell using an SPI 10 VMI mixer.

\subsubsection{Operating Process}

The kneading was performed using an SPI 10 mixer equipped with a rotating bowl, a central rod and an oblique shaft which was used for mixing the dough (Fig. 6). Experiments were performed at two constant mixing rates and two mixing times; $3 \mathrm{~min}$ at $100 \mathrm{rpm}$ for homogenization and 15 min kneading at $200 \mathrm{rpm}$ as maturation step.

The standard formulation (Table 1) was used as a reference with respect to the thermal coefficient of $54^{\circ} \mathrm{C}$ for all components (ambient temperature + flour temperature + water temperature $=54^{\circ} \mathrm{C}$ ).

The principal ingredients were incorporated in a specific order (flour, yeast and water). The addition of salt depended on the application. For some, salt was added to the standard mixture at the beginning and for others it was added after 5 minutes to estimate the change in texture on the acoustic properties.

\subsubsection{Results}

In order to understand the phenomena governing this part of the process (kneading phase), Fig. (7) illustrates the evolution of the acoustic amplitude of the signal response of the vibratory state of the rod (sensor). According to Kaddour [24], the curve shows the different steps before reaching a maximum value.

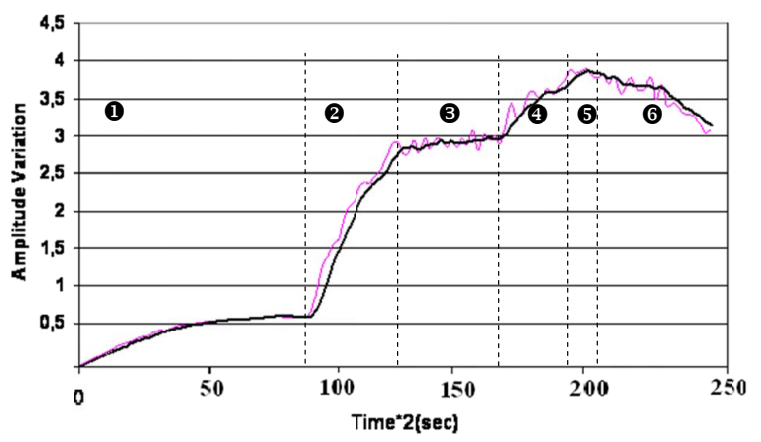

Fig. (7). Different steps govern the evolution of the physical state of the matter showing the critical points in the kneading phenomenon and the formation of the dough using the acoustic magnitudes.

The process can be divided into 6 steps:

(1) Homogenization: mixing of the raw materials at 100 rpm. Phase transition from a granular medium to an evolving bonded network.

(2) Gluten network development phase: starting at 200 rpm when the first phase reaches a constant value.

(3) Addition of salt affecting the equilibrium of matrix constitution. This phase is sullied by a brief decrease in the elasticity component of the matter. This decrease can be attributed to the decreases in interprotein hydrophobic interactions, which fall as a result of their effects on the water structure; these decreases reduce the tendency of proteins to aggregate, reducing momentarily elasticity before a new tendency for a matter relatively more consistent [6].

(4) Re-organization of the network and increase in the elasticity component resulting from the water pumping by the salt added.

(5) Process optimum: physical equilibrium of the network and the matrix components.

(6) Excess of mechanical energy leads to the destruction of the network followed by a decrease in the elasticity of the product.

The salt is then added at the same time as the other raw materials. The aim was to reduce the number of parameters varying during the process, as the curves in Fig. (8) particularly show that the two applications converge towards the same results. 


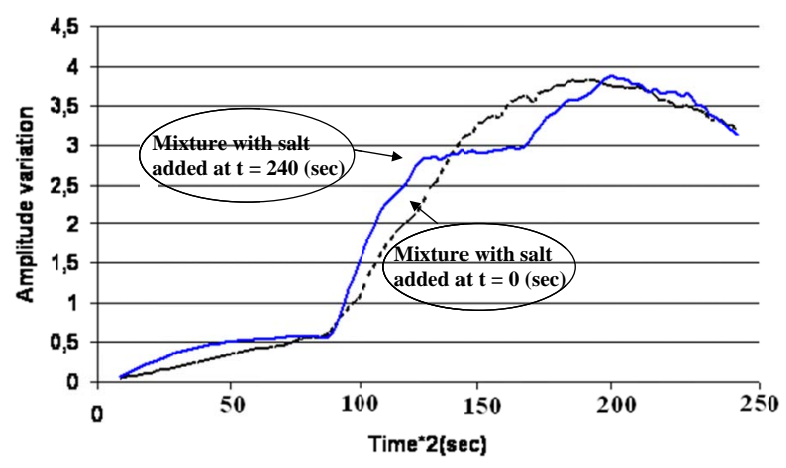

Fig. (8). Curves showing the same convergence of the physical state of two matters with varied salt addition:

- Dashed line: salt added at the beginning of process.

- $\quad$ Continuous line: salt added 9 min after the beginning.

By using the acoustic measurement technique for samples with different water content, from the "Max" and the shapes of the evolution curves in Fig. (9), it can be seen that there is a difference in the viscoelastic component according to the technological parameter applied. We can conclude that the extended shape of the "water less than 5\%" sample curve reflects higher mechanical resistance, and then more elastic in comparison with the others and the excess water gives a more viscous matter supporting the links in the gluten network. It is known that, the process gives underdeveloped dough in the case of lack of water or a very sticky product in the case of excess water.

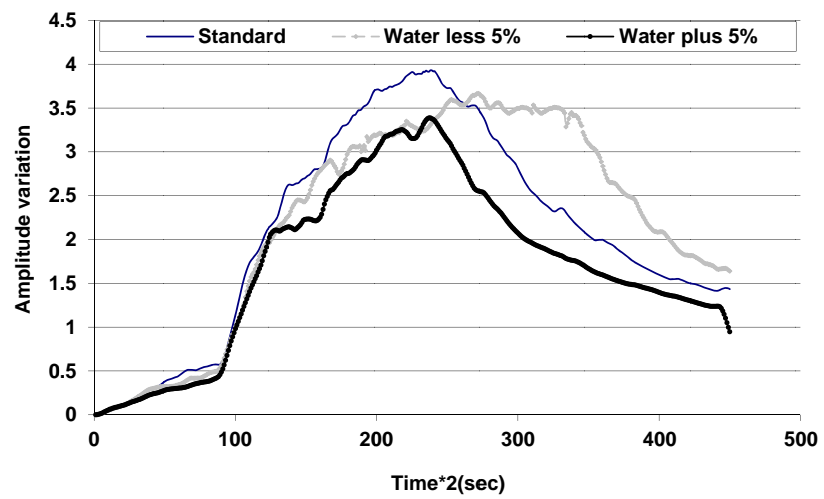

Fig. (9). Curves showing the difference in the viscoelastic behaviour of three samples with different water content.

According to the work of Farahnaky [25], the curves show the effect of added water on consistency. Added water softens the dough and decreases the hydration time and the energy required for mixing.

To quantify this evolution, we considered that stability was attained when this relative delay reached $95 \%$ of its maximum value (Fig. 10). An equilibrium phase $\boldsymbol{T}_{\boldsymbol{k}}$ resulting from the mechanical properties of the matrix was also defined during which the amplitude variation reached its maximum and remained stable.

Table 2 quantifies the acoustics parameters for the three samples having a difference in the water content.

The water content as a parameter in the process induces significant variation in the viscoelastic properties of the matter, especially in the stability phase. The higher elasticity of the "water less than 5\%" sample in the kneading phase, shown by the extension of the higher part of the curve, leads to a brittle matrix in the final product in comparison with the "water plus 5\%" sample. The latter definitely has greater extensible properties under the effect of the pressure of the gas produced in the matter. This phenomenon is marked by an early decrease (collapse step) on the curve after the max (internal mechanical equilibrium).

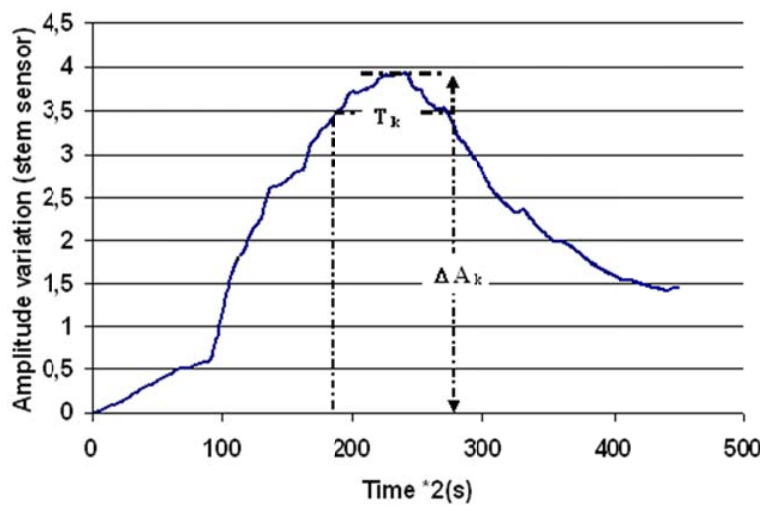

Fig. (10). Characteristic acoustic parameters defining some critical points in the evolution process.

Table 2. Quantification of the Maturity Component According to the Stability Time $T_{k}$ and the Amplitude Value $\Delta A_{k}$ of the Samples

\begin{tabular}{|c|c|c|c|}
\hline & $\mathbf{T}_{\mathbf{k}}$ & & $\Delta \mathbf{A}_{\mathbf{k}}$ \\
\hline \hline Water less 5\% & 106 & & 3,6 \\
\hline Standard & 50 & & \\
\hline Water plus 5\% & 43 & & 3,9 \\
\hline
\end{tabular}

Fig. (11) shows the differences between the mechanical parameters of three samples in comparison with a reference. In fact, by measuring the amplitude (flexion of the steam sensor) the effect of salt and olive oil on the elasticity component of the samples can be observed.

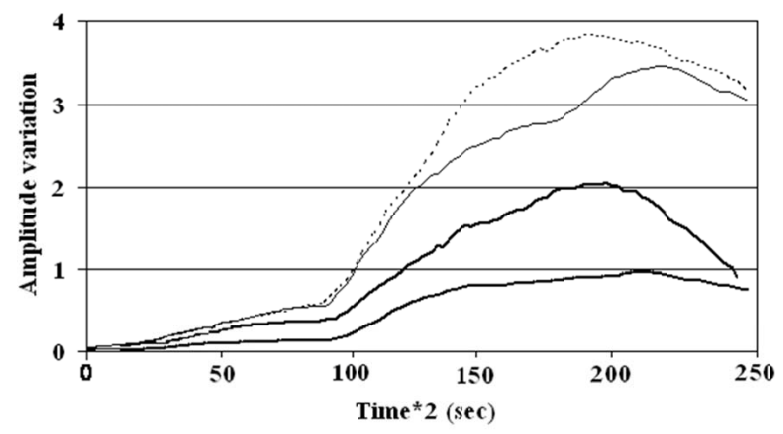

Fig. (11). Profile of the mechanical evolution of 4 dough samples according to the variation in acoustical amplitude; Reference: water + flour + salt; S1: Reference + olive oil $(10 \%)$; S2: water + flour; S3: water + flour + olive oil $(10 \%)$.

Table 3 provides an indicator, extrapolated from Fig. (11), of the consistency of the four samples (Reference, $\mathrm{S} 1, \mathrm{~S} 2, \mathrm{~S} 3$ ) at maturation (Max \& index). 
Table 3. Impact of the Additives on the Quality of the Final Product: Dough at Maturation

\begin{tabular}{|c|c|c|}
\hline & Max & Index (sec) \\
\hline \hline Reference & 3.9 & 362 \\
\hline S1 & 3.47 & 440 \\
\hline S2 & 1.97 & 380 \\
\hline S3 & 0,96 & 426 \\
\hline
\end{tabular}

The addition of salt at the start affects the global moisture component of the dough by water absorption, thus making the final product (dough) more elastic. The addition of oil affects the texture and consistency and makes the matter more "granular" while reducing elasticity. These results are in agreement with the literature $[25,26]$.

\section{CONCLUSION}

This work has examined the potential of two non-destructive acoustic methods to characterize the development of a complex matrix (dough kneading process). This technique based on passive acoustic sensors exploits the mechanical noise emitted by the process. The measurements characterize the phasetransition and define the critical points that govern the phenomenon. Following the development of the sensor technologies, it was shown that the signals received with this technique evolve differently according to the properties of the product used. The results from this technique appear to be sensitive to the different technological parameters in a complex process. Nevertheless, it appears that acoustic measurement through acoustic amplitude variation can potentially be used as an effective in-line quality control technique in viscoelastic complex media processes.

\section{CONFLICT OF INTEREST}

The authors confirm that this article content has no conflict of interest.

\section{ACKNOWLEDGEMENTS}

Declared none.

\section{REFERENCE}

[1] Hoseney RC. Glass transition and its role in cereals. In Principles of cereal science and technology. $2^{\text {nd }}$ ed. USA: American Association of Cereal Chemist 1998; pp. 307-20.

[2] Mirsaeedghazi H, Emam-Djomeh Z, Ali Mousavi M. Rheometric measurement of dough rheological characteristics and factors affecting. Int J Agric Biol 2008; 10: 112-9.

[3] Abdelrahman AA, Spies RD. Dynamic rheological studies of dough systems. In: Faridi H, Faubion JM, Eds. Fundamentals of dough rheology. USA: American Association of Cereal Chemist. MinnesotaL St. Paul 1986; pp. 87-103.

[4] Mani K, Eliasson AC, Lindahl L, Trägardh C. Rheological properties and breadmaking quality of wheat flour doughs made with different dough mixers. Cereal Chem 1992; 69: 222-5.
[5] Ait Kaddour A, Cuq B. Dynamic NIR spectroscopy to monitor bread dough mixing: a short review. Am J Food Technol 2011; 6(3): 173-85.

[6] Salvador A, Sanz T, Fiszman SM. Dynamic rheological characteristics of wheat flour-water doughs. Effect of adding $\mathrm{NaCl}$, sucrose and yeast. Food Hydrocoll 2006; 20(6): 780-6.

[7] Rajasree K, Vidialal V, Radhakrishnan P, Nampoori VPN, Vallabhan CPG. Use of mirage effect for the detection of phase transitions in solids. Meas Sci Technol 1993; 4(3): 435-7.

[8] Willias PR, Ravji S, Roberts ID. The pulse-resonance rheometer: an instrument for studying the viscoelastic properties of gels. Meas Sci Technol 2000; 11(4): 358-66.

[9] Hibberd GH. Dynamic viscoelastic behaviour of wheat flour doughs. Rheol Acta 1970; 9: 497-500.

[10] Smith JR, Smith TL, Tschoegl NW. Rheological properties of wheat flour doughs. III Dynamic shear modulus and its dependence on amplitude, frequency and dough composition. Rheol Acta 1970; 9: 239-52.

[11] Khatkar BS, Schofield JD. Dynimic rheology of wheat flour dough. I. Non-linear viscoelastic behavior. J Sci Food Agric 2002; 82: 827-9.

[12] Eliasson AC. Viscoelastic behaviour during the gelatinisation of starch. I. Comparison of wheat, maize, potatoand waxy-berlea starches. J Texture Stud 1986; 17: 253-65.

[13] Nassar G, Lefbvre F, Skaf A, Carlier J, Nongaillard B, Noêl Y. Ultrasonic and acoustic investigation of cheese matrix at the beginning and the end of ripening period. J Food Eng 2010; 96: 113.

[14] Kidmose U, Pedersen L, Nielsen M. Ultrasonics in evaluating rheological properties of dough from different wheat varieties and during ageing. J Texture Stud 2001; 32: 321-34.

[15] Lee HO, Luan H, Daut DG. Use of an ultrasonic technique to evaluate the rheological properties of cheese and dough. J Food Eng 1992; 16: 127-50.

[16] Létang C, Piau M, Verdier C. Characterization of wheat flourwater doughs. Part I: Rheometry and microstructure. J Food Eng 1999; 41: 121-32.

[17] Owolabi G M, Bassim MN, Page JH, Scanlon MG. The influence of specific mechanical energy on the ultrasonic characteristics of extruded dough. J Food Eng 2008; 86: 202-6.

[18] Ross KA, Pyrak-Nolte LJ, Campanella OH. The use of ultrasound and shear oscillatory tests to characterize the effect of mixing time on the rheological properties of dough. Food Res Int 2004; 37: 56777.

[19] Elmehdi HM, Page JH, Scanlon MG. Monitoring dough fermentation using acoustic waves. Trans Inst Chem Eng 2003; 81(C): 217-23.

[20] Janessen A. Obelisk and Katepwa wheat gluten, A study of factors determining bread-making performance. $\mathrm{Ph}$. D. thesis. Netherlands: Wageningen Agricultural University, 1992.

[21] Autio K, Laurikainen T. Relationships between flour/dough microstructure and dough handling and baking properties. Trends Food Sci Technol 1997; 8: 181-5.

[22] Chen WZ, Hoseney RC. Development of an objective method for dough stickiness. Lebensm Wiss Technol 1995; 28 (5): 467-73.

[23] Zheng H, Morgensternt MP, Campanella OH, Larsen NG. Rheological properties of dough during mechanical dough development. J Cereal Sci 2000; 32: 293-306.

[24] Kaddour AA, Barron C, Morel M-H, Cup B. Dynamic monitoring of dough mixing using near infrared spectroscopy: physical and chemical outcomes. Cereal Chem 2007; 84 (1): 70-9.

[25] Farahnaky A, Hill SE. The effect of salt, water and temperature on wheat dough rheology. J Texture Stud 2007; 38(4): 499-510.

[26] Mirsaeedghazi H, Emam-Djomeh Z, Mohamed Ali Mousavi S. Rheometric measurement of dough rheological characteristics and factors affecting it. Int J Agric Biol 2008; 10: 112-9. 